\title{
AGLR is a novel index for the prognosis of hepatocellular carcinoma patients: a retrospective study
}

Yan Liao ${ }^{1,2+}$, Rongyu Wei ${ }^{1 \dagger}$, Renzhi Yao ${ }^{1 \dagger}$, Liling Qin ${ }^{1}$, Jun $\mathrm{Li}^{1}$, Junxiong Yu ${ }^{3^{*}}$ and Weijia Liao ${ }^{1^{*}}$ (D)

\begin{abstract}
Background: Most hepatocellular carcinoma (HCC) patients'liver function indexes are abnormal. We aimed to investigate the relationship between (alkaline phosphatase + gamma-glutamyl transpeptidase)/lymphocyte ratio (AGLR) and the progression as well as the prognosis of HCC.

Methods: A total of $495 \mathrm{HCC}$ patients undergoing radical hepatectomy were retrospectively analyzed. We randomly divided these patients into the training cohort $(n=248)$ and the validation cohort $(n=247)$. In the training cohort, receiver operating characteristic (ROC) curve was used to determine the optimal cut-off value of AGLR for predicting postoperative survival of HCC patients, and the predictive value of AGLR was evaluated by concordance index (C-index). Further analysis of clinical and biochemical data of patients and the correlation analysis between AGLR and other clinicopathological factors were finished. Univariate and multivariate analyses were performed to identify prognostic factors for HCC patients. Survival curves were analyzed using the Kaplan-Meier method.
\end{abstract}

Results: According to the ROC curve analysis, the optimal predictive cut-off value of AGLR was 90 . The C-index of AGLR was 0.637 in the training cohort and 0.654 in the validation cohort, respectively. Based on this value, the HCC patients were divided into the low-AGLR group ( $A G L R \leq 90$ ) and the high-AGLR group ( $A G L R>90)$ ). Preoperative $A G L R$ level was positively correlated with alpha-fetoprotein (AFP), tumor size, tumor-node-metastasis (TNM) stage, and microvascular invasion (MVI) (all $p<0.05$ ). In the training and validation cohorts, patients with $A G L R>90$ had significantly shorter OS than patients with $A G L R \leq 90(p<0.001)$. Univariate and multivariate analyses of the training cohort $(\mathrm{HR}, 1.79 ; 95 \% \mathrm{Cl} 1.21-2.69 ; p<0.001)$ and validation cohort ( $\mathrm{HR}, 1.82 ; 95 \% \mathrm{Cl} 1.35-2.57 ; p<0.001)$ had identified AGLR as an independent prognostic factor. A new prognostic scoring model was established based on the independent predictors determined in multivariate analysis.

Conclusions: The elevated preoperative AGLR level indicated poor prognosis for patients with HCC; the novel prognostic scoring model had favorable predictive capability for postoperative prognosis of HCC patients, which may bring convenience for clinical management.

Keywords: Hepatocellular carcinoma, AGLR, Prognosis, Biomarker

*Correspondence: yujunxiong2897320@163.com; liaoweijia288@163.com

†Yan Liao, Rongyu Wei and Renzhi Yao contributed equally to this work ${ }^{1}$ Laboratory of Hepatobiliary and Pancreatic Surgery, Affiliated Hospital of Guilin Medical University, Guilin 541001, Guangxi, People's Republic of China

${ }^{3}$ Department of Anesthesiology, The Second Affiliated Hospital of Guilin Medical University, Guilin 541001, Guangxi, People's Republic of China Full list of author information is available at the end of the article

\section{Background}

Cancer is a significant threat to public health worldwide, and the incidence rate of hepatocellular carcinoma (HCC) has been in a rising trend in recent years [1]. Southeast Asia and sub-Saharan Africa are the high distribution regions of HCC, where chronic hepatitis B virus 
(HBV) infection is prevalent [2]. Despite the considerable improvement on HCC diagnosis, advancement in surgical resection and liver transplantation in clinical practice, the prognosis of postoperative HCC patients remains unsatisfactory due to the high metastasis and recurrence rates. Therefore, researches on the critical factors affecting prognosis of liver cancer are of great significance to improve the therapeutic efficacy of HCC patients, and promote patient management.

Unlike other cancers, the prognosis of HCC depends not only on tumor malignancy, but also on the remaining liver function. Liver function test is a routine biochemical test used to evaluate liver dysfunction. Alkaline phosphatase (ALP) and gamma-glutamyl transpeptidase (GGT) are the representative enzymes in serum, as well as the parameters for liver function. Previous studies have reported that ALP, GGT and lymphocyte count were independent prognostic predictors for liver cancer [3-5]; and ALP to lymphocyte count ratio or GGT to lymphocyte count ratio could serve as prognostic factors as well $[5,6]$. It was found that, the normal references of serum ALP and GGT level were roughly equal in clinical, and a complementary effect was speculated between these two factors; meanwhile, the limitation of a single factor for predicting HCC prognosis should be considered. Therefore, it was assumed that a parameter composed of the two factors may have more favorable prognostic predictive capacity, and a prognosis prediction model made up of multiple factors was constructed: [ALP (U/L) + GGT $(\mathrm{U} / \mathrm{L})] /$ lymphocyte count $\left(\times 10^{9} / \mathrm{L}\right)$ (AGLR), and this model may have great potential for postoperative prognosis prediction for HCC patients.

\section{Methods}

\section{Patients}

495 HCC patients undergoing surgical resection at the Affiliated Hospital of Guilin Medical University (Guilin, People's Republic of China) from February 2005 to December 2012 conformed to the inclusion criteria of this study. The pathologic examination of $\mathrm{HCC}$ was implemented based on the Primary Liver Cancer Clinical Diagnosis and Staging Criteria (Ministry of Health, Beijing, China). The baseline information includes: (1) demographics characteristics: age, gender, drinking, etc.; (2) preoperative laboratory tests: hepatitis B surface antigen (HBsAg), alpha-fetoprotein (AFP), aspartate transaminase, alanine aminotransferase, ALP, GGT, etc.; (3) tumor characteristics: combined with liver cirrhosis, the size and the number of tumors, clinical tumor node metastasis (TNM) stage, microvascular invasion (MVI), recurrence after radical resection, etc. Patients who lost contact during follow-up or with incomplete data were excluded. All methods were carried out abode by the
Affiliated Hospital of Guilin Medical University's guidelines and regulations. This study was approved by the research ethics committee of the Affiliated Hospital of Guilin Medical University and complied with the Declaration of Helsinki Principles. Informed consents were obtained from all patients.

Postoperative long-term follow-up included serum AFP level and abdominal ultrasonography every two months and chest radiography every six months in the first 2 years and at 3 - and 6-month intervals respectively after that. Patients would undergo computerized tomography or magnetic resonance imaging scan if recurrence was suspected [7]. Overall survival (OS) was defined as the time from the date of surgery to the date of death or the last follow-up. Disease-free survival (DFS) refers to the time from radical resection to recurrence, metastasis, death or the last follow-up.

\section{Statistical analysis}

All statistical analyses were performed using SPSS 18.0 (SPSS Inc, Chicago, IL) and R (version 3.6.1, R Foundation for Statistical Computing, Vienna, Austria. URL https://www.R-project.org/). The receiver operating characteristic (ROC) curve was used to analyze and calculate the area under the curve (AUC), and the optimal cut-off value was determined by calculating the largest Youden index (sensitivity + specificity-1). Concordance index was determined to predict probability that predicted results were in accordance with the actual results, and C-index greater than 0.5 suggested a certain predictive value of this model. Continuous variables conforming to the normal distribution were expressed as mean \pm standard deviation (SD). The comparison of categorical variables was evaluated using the Chi-square test. Kaplan-Meier statistics and Log rank test were used to analyze the different clinical factors related to survival. According to the Cox proportional hazard model, multivariate analysis was performed by SPSS 18.0 (SPSS Inc, Chicago, IL) to explore the independent prognostic value of variables with significance in univariate analysis, and the hazard ratio (HR) and 95\% confidence interval (95\% CI) were calculated. The survival curves were performed using the Kaplan-Meier method, and the statistical difference of survival distributions between different groups was compared using the log-rank test. Statistical significance was considered if $p<0.05$.

\section{Results}

\section{Clinical and biochemical data}

We recruited 495 HCC patients and randomly divided them into the training cohort (248 patients) and the validation cohort (247 patients). The mean postoperative follow-up time was 51.6 months (median, 46.0 months; 
range, 2.0 to 120.0 months). In the training and validation cohorts, the median age of patients was 49.33 and 50.96 years, respectively. The proportion of male patients was much higher than that of female patients, and there were 219 male cases $(88.3 \%)$ in the training cohort and 213 male cases $(86.2 \%)$ in the validation cohort, which may be caused by the higher proportion of male liver cancer patients in Asian countries. Clinical and biochemical data were further statistically compared between the training and validation cohorts. The results were shown in Table 1.

\section{The relationship between preoperative AGLR level} and clinical pathologic characteristics in patients with HCC Using the receiver operator characteristics (ROC) analysis, the optimal predictive cut-off value of AGLR was 90 , with the sensitivity of $75.1 \%$, the specificity of $64.8 \%$ and the area under the curve (AUC) was 0.735 (95\% CI 0.679-0.786), according to the postoperative survival of HCC patients in the training cohort. Based on this cutoff value, our patients could be divided into two groups by dichotomy: AGLR $\leq 90$ and AGLR $>90$ groups. Given that serum AFP level is a prognostic factor of liver cancer, and neutrophil-to-lymphocyte ratio (NLR) and albumin bilirubin (ALBI) grade are also common indicators for monitoring the prognosis of liver cancer, we used the same method to calculate the AUCs of AFP, NLR and ALBI, respectively. Interestingly, it was revealed that the AUCs of AGLR were higher than that of AFP, NLR and ALBI in both training cohort and validation cohort (Figs. 1a, 2a). Meanwhile, C-index of AGLR suggested that both AGLR (C-index $=0.637,95 \%$ CI $0.597-0.684$ ) and AFP (C-index $=0.624,95 \%$ CI $0.585-0.671$ ) had predictive value in the training cohort, and more importantly, AGLR had a higher accuracy than AFP; and the value of AGLR (C-index $=0.654,95 \%$ CI $0.613-0.707$ ) and AFP (C-index $=0.577,95 \%$ CI $0.532-0.633$ ) were both verified in the validation cohort. The relationships between preoperative AGLR level and clinicopathologic characteristics were investigated and results were shown in Table 2. In the training cohort (248 patients), high preoperative AGLR level was positively correlated with serum AFP level $(>20 \mathrm{ng} / \mathrm{ml})(p<0.001)$, tumor size $>5 \mathrm{~cm}(p<0.001)$, multiple tumors $(p=0.035)$, TNM stage III-IV $(p<0.001)$, presence of MVI $(p<0.001)$. And in the validation cohort (247 patients), high preoperative AGLR level was positively correlated with serum AFP level $(>20 \mathrm{ng} / \mathrm{ml})(p<0.001)$, tumor size $>5 \mathrm{~cm}$ $(p<0.001)$, TNM stage III-IV $(p<0.001)$, presence of MVI $(p<0.001)$. However, there were no obvious correlations between AGLR $>90$ and age, gender, drinking, HBsAg, liver cirrhosis and recurrence (all $p>0.05$ ). Moreover, higher AGLR level was found in tumor size $>5 \mathrm{~cm}$, TNM stage III-IV and MVI patients ( $p<0.05$, Figs. $1 \mathrm{~b}, 2 \mathrm{~b}$ ).

\section{Survival analysis based on different preoperative AGLR levels}

In the training cohort, the average survival time for DFS patients with AGLR $\leq 90$ was 77.42 months (95\% CI 67.70-87.13), and for DFS patients with AGLR >90, the average survival time was 39.52 months (95\% CI 31.90-47.15) ( $p<0.001$, Fig. 3a). Among OS patients, the average survival time of patients with AGLR $\leq 90$ was 83.60 months (95\% CI 75.18-92.03) and the 1-, 3and 5-year survival rates were $86.3 \%, 71.6 \%$ and $63.1 \%$, respectively; while for AGLR > 90 patients, they had an average OS of 47.39 months (95\% CI 40.26-54.53) and the 1-, 3- and 5-year survival rates were $77.6 \%, 44.8 \%$ and $27.4 \%$, respectively ( $p<0.001$, Fig. $3 \mathrm{~b})$.

In the validation cohort, the average survival time for DFS patients with AGLR $\leq 90$ was 75.58 months $(95 \%$ CI 66.12-85.03), and for patients with AGLR $>90$, the average survival time was 49.28 months (95\% CI 41.42-57.14) $(p<0.001$; Fig. 4a). In OS patients, for HCC patients with AGLR $\leq 90$, the average survival time was 83.66 months (95\% CI 75.65-91.66) and the 1-, 3- and 5-year survival rates were $88.7 \%, 73.0 \%$ and $60.8 \%$, respectively; and for patients whose AGLR $>90$, they had a mean OS of 59.30 months (95\% CI 52.10-66.50) and the 1-, 3and 5 -year survival rates were $73.5 \%, 46.9 \%$ and $36.1 \%$, respectively $(p<0.001$, Fig. 4 b) .

\section{Prognostic factors of survival for patients with HCC}

The Cox univariate and multivariate regression analyses were applied to evaluate the prognostic value of AGLR and other factors. In the training cohort, it was found that AGLR $>90(\mathrm{HR}=1.79,95 \%$ CI $1.21-2.69, p<0.001)$, tumor size $(\mathrm{HR}=1.91,95 \%$ CI $1.27-2.61, p<0.001)$, TNM stage $(\mathrm{HR}=1.52,95 \%$ CI $1.03-2.31, p=0.025)$, MVI $(\mathrm{HR}=1.61,95 \% \mathrm{CI} 1.23-2.39, p=0.007)$ and recurrence $(\mathrm{HR}=2.01,95 \% \mathrm{CI} 1.47-2.83, p<0.001)$ were five crucial independent predictors of OS for HCC patients (Table 3), and similar result was found in the validation cohort either (Table 4).

Then, each of the above five independent predictors were assigned, such as AGLR $\leq 90$ was assigned 0 point and AGLR $>90$ was assigned 1 point, and other four predictors were assigned in the same manner. Thus, all HCC patients would be divided into six groups of different scores, ranging from 0 to 5 points, based on their accumulated total scores. As the result, a new prognostic scoring model consisted of multiple variables was constructed. However, some comparisons between two of these new groups had no statistical different. For instance, in the training cohort, for 
Table 1 Clinical and biochemical data of examined patients

\begin{tabular}{|c|c|c|c|}
\hline Parameter & $\begin{array}{l}\text { Training cohort } \\
(n=248)\end{array}$ & $\begin{array}{l}\text { Validation cohort } \\
(n=247)\end{array}$ & $p$ value \\
\hline \multicolumn{4}{|l|}{ Basic information } \\
\hline Age (years) & $49.33 \pm 11.35$ & $50.96 \pm 11.80$ & 0.119 \\
\hline \multicolumn{4}{|l|}{ Gender: $\mathrm{n}(\%)$} \\
\hline Female & $29(11.7)$ & $34(13.8)$ & 0.489 \\
\hline Male & $219(88.3)$ & $213(86.2)$ & \\
\hline \multicolumn{4}{|l|}{ Family history: n (\%) } \\
\hline No & $216(87.0)$ & $219(88.7)$ & 0.435 \\
\hline Yes & $32(13.0)$ & $28(11.3)$ & \\
\hline \multicolumn{4}{|l|}{ Drinking: n (\%) } \\
\hline No & $140(56.5)$ & $133(53.8)$ & 0.560 \\
\hline Yes & $108(43.5)$ & $114(46.2)$ & \\
\hline \multicolumn{4}{|l|}{ Smoking: n (\%) } \\
\hline No & $148(59.7)$ & $152(61.5)$ & 0.577 \\
\hline Yes & $100(40.3)$ & $95(38.5)$ & \\
\hline \multicolumn{4}{|l|}{ HBsAg: n (\%) } \\
\hline Negative & $41(16.5)$ & $34(13.8)$ & 0.391 \\
\hline Positive & $207(83.5)$ & $213(86.2)$ & \\
\hline \multicolumn{4}{|l|}{ Minimally invasive surgery: $\mathrm{n}(\%)$} \\
\hline No & $184(74.2)$ & $180(72.9)$ & 0.739 \\
\hline Yes & $64(25.8)$ & $67(27.1)$ & \\
\hline \multicolumn{4}{|l|}{ Lab check data } \\
\hline WBC $\left(\times 10^{9} / \mathrm{L}\right)$ & $6.04 \pm 2.01$ & $6.39 \pm 2.20$ & 0.061 \\
\hline NEUT $\left(\times 10^{9} / \mathrm{L}\right)$ & $3.65 \pm 1.71$ & $3.92 \pm 1.73$ & 0.081 \\
\hline $\mathrm{LYMPH}\left(\times 10^{9} / \mathrm{L}\right)$ & $1.64 \pm 0.57$ & $1.73 \pm 0.65$ & 0.105 \\
\hline Platelets $\left(\times 10^{9} / \mathrm{L}\right)$ & $173.96 \pm 75.18$ & $181.23 \pm 79.27$ & 0.096 \\
\hline Albumin (g/L) & $39.07 \pm 4.59$ & $39.65 \pm 4.66$ & 0.220 \\
\hline Globulin (g/L) & $31.01 \pm 5.80$ & $30.35 \pm 6.18$ & 0.215 \\
\hline TBIL $(\mu \mathrm{mol} / \mathrm{L})$ & $15.91 \pm 14.33$ & $16.45 \pm 16.07$ & 0.747 \\
\hline $\mathrm{DBIL}(\mu \mathrm{mol} / \mathrm{L})$ & $6.33 \pm 12.38$ & $6.92 \pm 13.17$ & 0.689 \\
\hline ALT (U/L) & $45.12 \pm 42.93$ & $51.08 \pm 46.33$ & 0.783 \\
\hline AST (U/L) & $49.98 \pm 48.83$ & $51.91 \pm 57.49$ & 0.697 \\
\hline $\operatorname{ALP}(U / L)$ & $95.69 \pm 65.26$ & $92.29 \pm 42.60$ & 0.493 \\
\hline GGT (U/L): median, range & $67.62,10.7-335.1$ & $72.19,10.0-351.76$ & 0.854 \\
\hline AGLR level: median, range & $90.63,19.43-441.72$ & $88.83,16.07-462.16$ & 0.521 \\
\hline AFP (ng/mL): median, range & $246.7,0.20-32,800$ & $220.7,0.60-25,410$ & 0.363 \\
\hline \multicolumn{4}{|l|}{ Pathological features } \\
\hline \multicolumn{4}{|l|}{ Cirrhosis: n (\%) } \\
\hline No & $24(10.0)$ & $13(5.3)$ & 0.062 \\
\hline Yes & $224(90.0)$ & $234(94.7)$ & \\
\hline Tumor size (cm) & $7.81 \pm 4.68$ & $7.12 \pm 4.11$ & 0.085 \\
\hline \multicolumn{4}{|l|}{ Tumor number: n (\%) } \\
\hline Single & $190(76.6)$ & $188(76.1)$ & 0.896 \\
\hline Multiple & $58(23.4)$ & $59(23.9)$ & \\
\hline \multicolumn{4}{|l|}{ TNM stage: n (\%) } \\
\hline$|-| \mid$ & $136(54.8)$ & $124(50.2)$ & 0.302 \\
\hline III-IV & $112(45.2)$ & $123(49.8)$ & \\
\hline \multicolumn{4}{|l|}{ MVI: n (\%) } \\
\hline No & $201(81.0)$ & $188(76.1)$ & 0.181 \\
\hline Yes & $47(19.0)$ & $59(23.9)$ & \\
\hline \multicolumn{4}{|l|}{ Recurrence: n (\%) } \\
\hline No & $158(63.7)$ & $148(59.9)$ & 0.385 \\
\hline Yes & $90(36.3)$ & $99(40.1)$ & \\
\hline
\end{tabular}


Table 1 (continued)

$\mathrm{N}$, number of patients; $\mathrm{HBsAg}$, hepatitis B surface antigen; WBC, white blood cell; LYMPH, lymphocyte count; TBIL, total bilirubin; DBIL, direct bilirubin; ALT, alanine aminotransferase; AST, aspartate aminotransferase; ALP, alkaline phosphatase; GGT, gamma-glutamyl transpeptidase; AGLR, ALP plus GGT to LYMPH; AFP, alphafetoprotein; TNM, tumor-node-metastasis; MVI, microvascular invasion
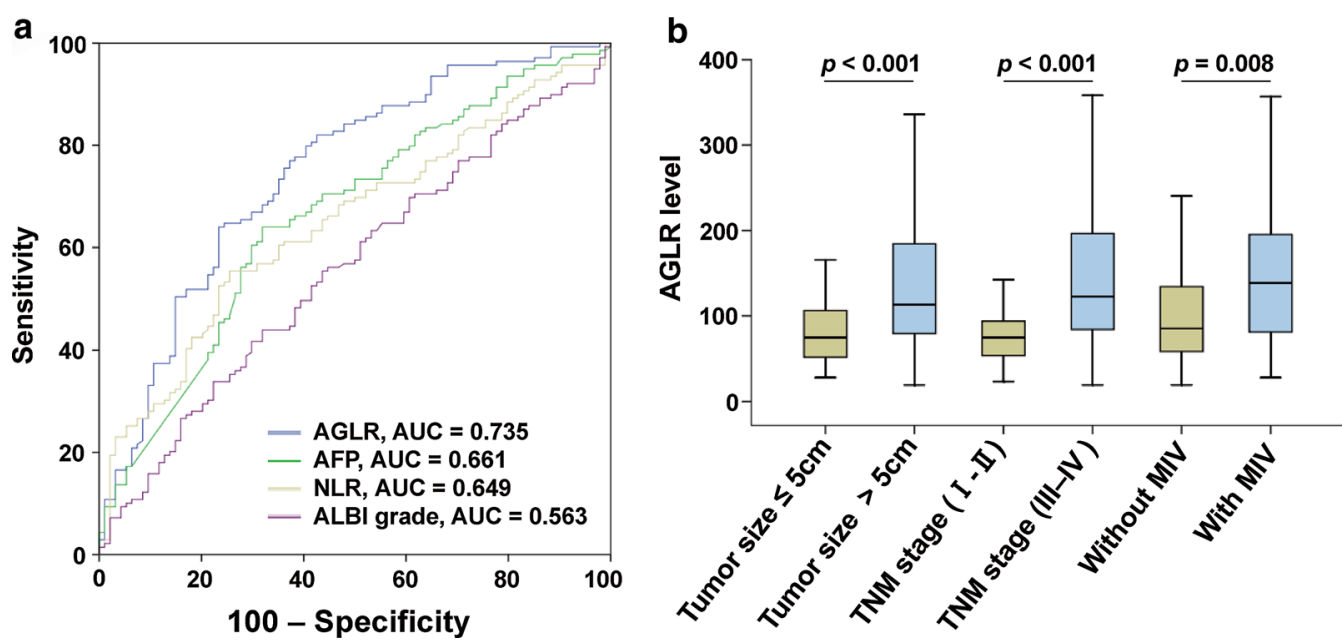

Fig. 1 AGLR's predictive capability and its comparison with AFP, NLR and ALBI grade. ROC of AGLR in the training cohort (a) and the relationships between AGLR level and tumor size, TNM stage and MVI in the training cohort (b)
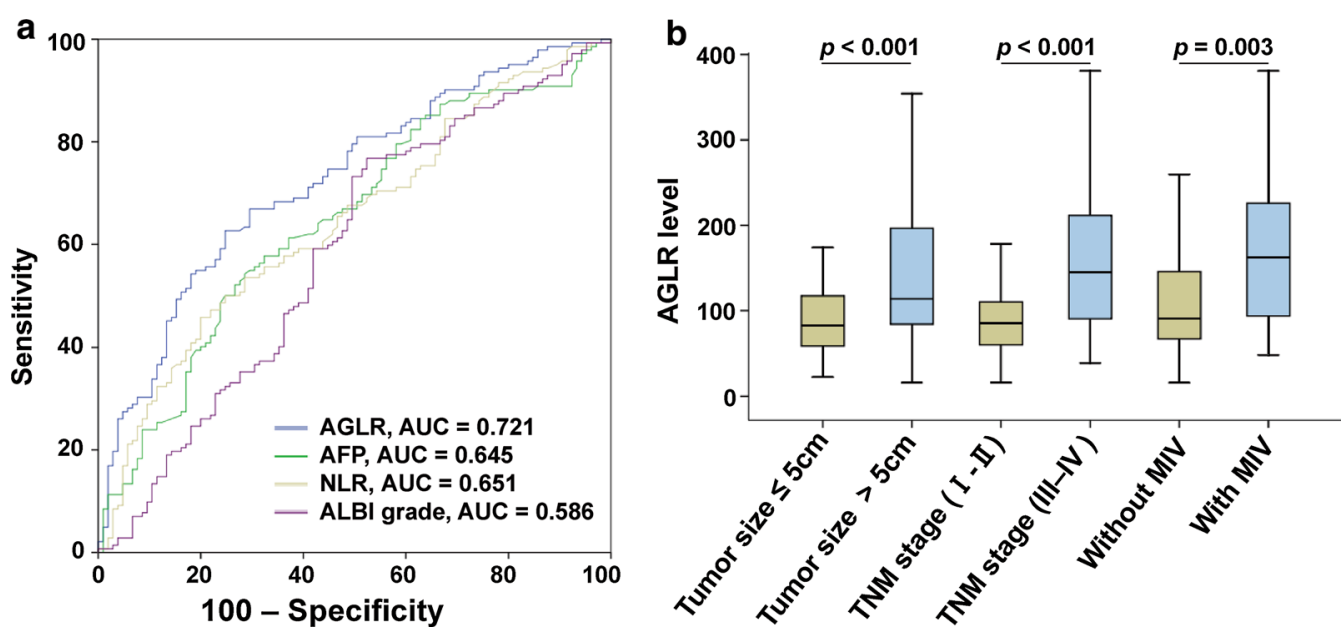

Fig. 2 ROC of AGLR in the validation cohort (a) and the relationships between AGLR level and tumor size, TNM stage and MVI in the validation cohort (b)

DFS patients with a score of 2 vs. $3(p=0.173)$ (Fig. $5 \mathrm{a}$ ) and for OS patients with a score of 2 vs. $3(p=0.126)$, score 3 vs. $4(p=0.062)$ and score 4 vs. $5(p=0.079)$ (Fig. 5b). Similar result was also found in the validation cohort (Fig. 6a, b). In view of these circumstances and in order to obtain better application value of this new model, we further divided these groups according to the scores: $0-1$ points (low-risk group), $2-3$ points (medium-risk group) and 4-5 points (high-risk group). Surprisingly, the survival analyses revealed that in both training cohort (Fig. 5c, d) and validation cohort (Fig. 6c, d), HCC patients' postoperative survival time had significant differences between the low-, mediumand high-risk groups, which was an obviously decreasing trend. 
Table 2 Correlation between clinical pathologic characteristics and AGLR level in HCC patients

\begin{tabular}{|c|c|c|c|c|c|c|}
\hline \multirow[t]{3}{*}{ Variables } & \multicolumn{6}{|l|}{ AGLR level } \\
\hline & \multicolumn{3}{|c|}{ Training cohort $(n=248)$} & \multicolumn{3}{|c|}{ Validation cohort $(n=247)$} \\
\hline & $\leq 90 \mathrm{n}(\%)$ & $>90 \mathrm{n}(\%)$ & $p$ value & $\leq 90 \mathrm{n}(\%)$ & $>90 \mathrm{n}(\%)$ & $p$ value \\
\hline \multicolumn{7}{|l|}{ Age (years) } \\
\hline$\leq 60$ & $92(46.2)$ & $107(53.8)$ & 0.347 & $81(41.3)$ & $115(58.7)$ & 0.815 \\
\hline$>60$ & $19(38.8)$ & $30(61.2)$ & & $22(43.1)$ & $29(56.9)$ & \\
\hline \multicolumn{7}{|l|}{ Gender } \\
\hline Female & $18(60.0)$ & $12(40.0)$ & 0.073 & $18(52.9)$ & $16(47.1)$ & 0.152 \\
\hline Male & $93(42.7)$ & $125(57.3)$ & & $85(39.9)$ & $128(60.1)$ & \\
\hline \multicolumn{7}{|l|}{ Drinking } \\
\hline No & $62(44.6)$ & $77(55.4)$ & 0.958 & $58(43.3)$ & $76(56.7)$ & 0.583 \\
\hline Yes & $49(45.0)$ & $60(55.0)$ & & $45(39.8)$ & $68(60.2)$ & \\
\hline \multicolumn{7}{|l|}{$\mathrm{HBsAg}$} \\
\hline Negative & $14(38.9)$ & $22(61.1)$ & 0.444 & $17(43.6)$ & $22(56.4)$ & 0.794 \\
\hline Positive & $97(45.8)$ & $115(54.2)$ & & $86(41.3)$ & $122(58.7)$ & \\
\hline \multicolumn{7}{|c|}{$\operatorname{AFP}(n g / m L)$} \\
\hline$\leq 20$ & $54(62.8)$ & $32(37.2)$ & $<0.001$ & $43(58.6)$ & $30(41.4)$ & $<0.001$ \\
\hline$>20$ & $57(35.2)$ & $105(64.8)$ & & $60(34.5)$ & $114(65.5)$ & \\
\hline \multicolumn{7}{|c|}{ Liver cirrhosis } \\
\hline No & $8(57.1)$ & $6(42.9)$ & 0.337 & $10(43.5)$ & $13(56.5)$ & 0.856 \\
\hline Yes & $103(44.0)$ & $131(56.0)$ & & $93(41.5)$ & $131(58.5)$ & \\
\hline \multicolumn{7}{|c|}{ Tumor size (cm) } \\
\hline$\leq 5$ & $66(65.3)$ & $35(34.7)$ & $<0.001$ & $61(56.5)$ & $47(43.5)$ & $<0.001$ \\
\hline$>5$ & 45 (30.6) & $102(69.4)$ & & $42(30.2)$ & $97(69.8)$ & \\
\hline \multicolumn{7}{|c|}{ Tumor number } \\
\hline Single & 90 (48.6) & $95(51.4)$ & 0.035 & $84(43.5)$ & $109(56.5)$ & 0.272 \\
\hline Multiple & $21(33.3)$ & $42(66.7)$ & & $19(35.2)$ & $35(64.8)$ & \\
\hline \multicolumn{7}{|l|}{ TNM stage } \\
\hline$|-| \mid$ & $82(68.9)$ & $37(31.1)$ & $<0.001$ & $81(57.4)$ & $60(42.6)$ & $<0.001$ \\
\hline III- IV & $29(22.5)$ & $100(77.5)$ & & $22(20.8)$ & $84(79.2)$ & \\
\hline \multicolumn{7}{|c|}{ Microvascular invasion } \\
\hline No & $95(52.2)$ & $87(47.8)$ & $<0.001$ & $96(46.2)$ & $112(53.8)$ & 0.001 \\
\hline Yes & $16(24.2)$ & $50(75.8)$ & & $7(17.9)$ & $32(82.1)$ & \\
\hline \multicolumn{7}{|l|}{ Recurrence } \\
\hline No & $70(47.0)$ & $79(53.0)$ & 0.388 & $71(45.2)$ & $86(54.8)$ & 0.138 \\
\hline Yes & $41(41.4)$ & $58(58.6)$ & & $32(35.6)$ & $58(64.4)$ & \\
\hline
\end{tabular}

AGLR, alkaline phosphatase plus gamma-glutamyl transpeptidase to lymphocyte ratio; HBsAg, hepatitis B surface antigen; AFP, alpha-fetoprotein; TNM, tumor-nodemetastasis

\section{Discussion}

In this study, we established a simple and evidence-based prognostic model, named AGLR, in order to predict the risk of survival for HCC patients undergoing radical resection, which incorporated routinely available laboratory parameters: ALP, serum GGT level and lymphocyte count. And this prognostic model was repeatable and accurate. Several studies have shown that elevated serum ALP level may be related to some pathological conditions [8-10], and other studies have revealed that ALP was a cancer-associated serum enzyme [11-13]. Moreover, according to the electron microscopic cytochemistry, ALP was observed to contain the nuclear localization signal and was linked to the proliferation of cancer cells [14]. Therefore, the elevation of serum ALP may play an essential role in cancer proliferation. GGT is an ubiquitous epithelial enzyme that associated with higher mortality in many diseases, including liver disease, pancreatic disease, renal failure, myocardial infarction and diabetes [15]. Lymphocytes may play an important role in immune 

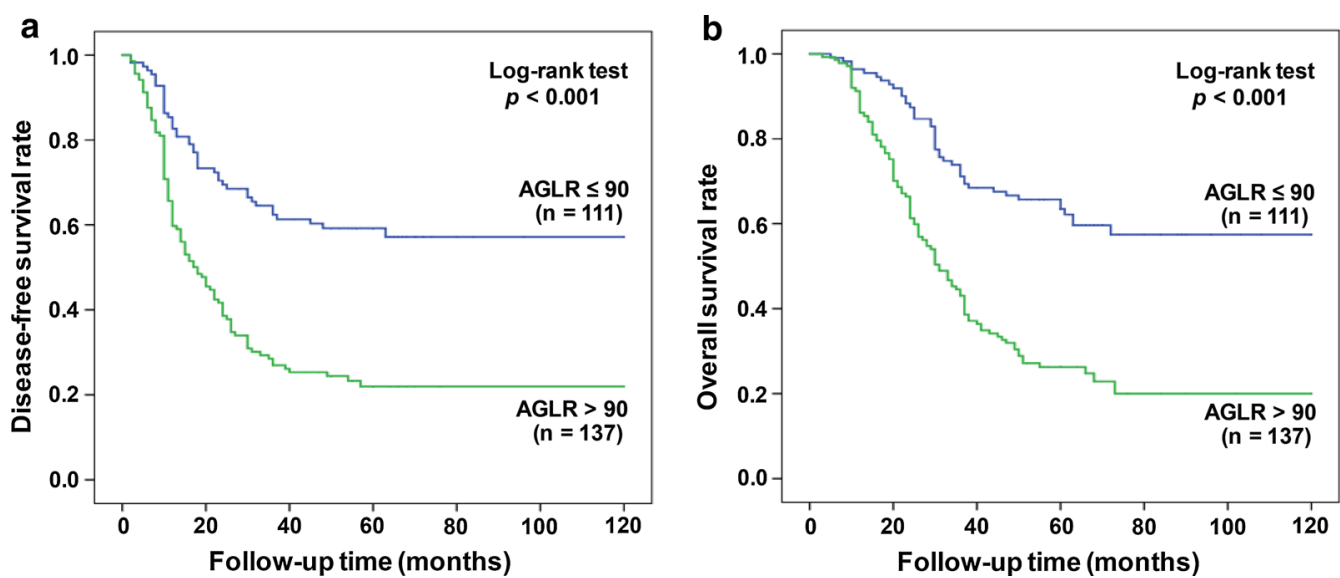

Fig. 3 Prognostic significance of AGLR in patients with HCC. Kaplan-Meier analysis of survival in the training cohort. High AGLR level was closely associated with a worse prognosis. The green line represents AGLR level $>90$, whereas the blue line represents AGLR level $\leq 90$. Kaplan-Meier curves depict OS (a) and DFS (b) in HCC patients with AGLR $>90$ or $\leq 90$
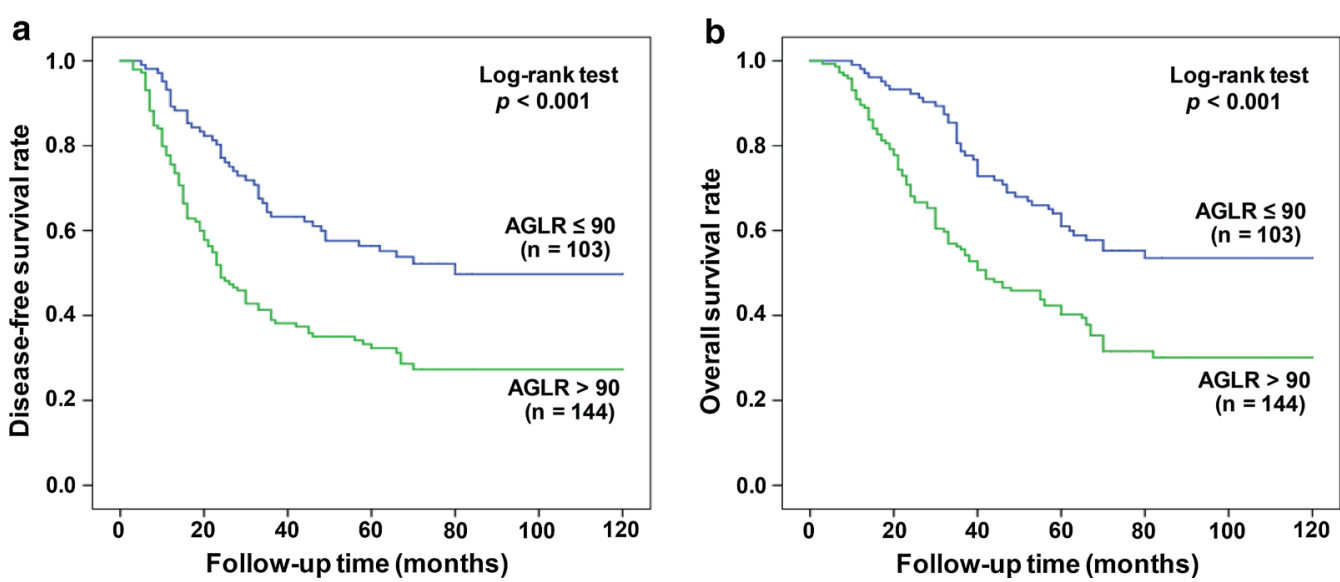

Fig. 4 Kaplan-Meier analysis of HCC patients' survival in the validation cohort. High AGLR level was closely associated with a worse prognosis. Kaplan-Meier curves depict OS (a) and DFS (b) in HCC patients with AGLR>90 or $\leq 90$

regulation of tumor; $\mathrm{T}$ cells could be activated by phytohemagglutinin (PHA), ionomycin (Iono) and other factors; in the meanwhile, $\mathrm{T}$ cells could be induced to apoptosis through a variety of ways [16]. Moreover, some researches revealed that reduced $\mathrm{CD} 8^{+} \mathrm{T}$ lymphocytes might have relation to unfavorable prognosis of liver cancer $[17,18]$. Therefore, all the three factors mentioned above are adverse factors for HCC patients; if all of them can be taken into consideration when predicting liver cancer patients' postoperative prognosis, more reliable prediction and preciser medical treatment will realize.

In this retrospective study, we first analyzed the clinical and biochemical data of training cohort and validation cohort, as well as the relationship between preoperative AGLR level and clinical characteristics of patients with
HCC. It is noteworthy that elevated preoperative AGLR level is positively related to tumor size $>5 \mathrm{~cm}$, TNM stage III-IV and MVI. This result was further confirmed in the validation cohort. However, MVI, as a unique way for HCC cells to invade the blood vessel, depends on the invasive and metastasizing potential of liver cancer cells. Therefore, it was speculated that elevated AGLR level may endow cancer cells with the possibility of invasion and metastasis through changing its micro-environment for metabolism, and further lead to the deterioration of HCC.

In addition to AGLR, AFP, tumor size, TNM stage, MVI and recurrence were also associated with a shorter OS for HCC patients. Previous studies have found that AFP promoted the invasion and metastasis of $\mathrm{HCC}$ 
Table 3 Univariate and multivariate analysis of overall survival (training cohort, $n=248$ )

\begin{tabular}{|c|c|c|c|c|}
\hline \multirow[t]{2}{*}{ Clinical character } & \multicolumn{2}{|c|}{ Univariate analysis } & \multicolumn{2}{|c|}{ Multivariate analysis } \\
\hline & HR $(95 \% \mathrm{Cl})$ & $p$ value & HR $(95 \% \mathrm{Cl})$ & $p$ value \\
\hline AGLR level $(>90$ vs $\leq 90)$ & $2.66(2.01-3.88)$ & $<0.001$ & $1.79(1.21-2.69)$ & $<0.001$ \\
\hline Age, years (> 60 vs $\leq 60)$ & $1.24(0.81-1.83)$ & 0.308 & & \\
\hline Gender (male vs female) & $1.23(0.72-1.91)$ & 0.441 & & \\
\hline Drinking (yes vs no) & $1.03(0.74-1.41)$ & 0.862 & & \\
\hline HBsAg (positive vs negative) & $1.29(0.78-2.06)$ & 0.303 & & \\
\hline AFP, ng/ml (> 20 vs $\leq 20)$ & $1.71(1.19-2.47)$ & 0.003 & $1.13(0.77-1.67)$ & 0.514 \\
\hline Liver cirrhosis (yes vs no) & $1.03(0.51-2.09)$ & 0.930 & & \\
\hline Tumor size, $\mathrm{cm}$ ( $>5$ vs $\leq 5)$ & $2.86(1.97-3.91)$ & $<0.001$ & $1.91(1.27-2.61)$ & $<0.001$ \\
\hline Tumor number (multiple vs single) & $1.60(1.13-2.26)$ & 0.006 & $1.12(0.81-1.53)$ & 0.460 \\
\hline TNM stage (III-IV vs I-II) & $1.96(1.39-2.77)$ & $<0.001$ & $1.52(1.03-2.31)$ & 0.025 \\
\hline MVI (yes vs no) & $2.57(1.93-3.74)$ & $<0.001$ & $1.61(1.23-2.39)$ & 0.007 \\
\hline Recurrence (yes vs no) & $2.70(1.69-3.59)$ & $<0.001$ & $2.01(1.47-2.83)$ & $<0.001$ \\
\hline
\end{tabular}

$\mathrm{Cl}$, confidence interval; $\mathrm{HR}$, hazard ratio; $\mathrm{AGLR}$, alkaline phosphatase plus gamma-glutamyl transpeptidase to lymphocyte ratio; $\mathrm{HBs} \mathrm{Ag}$, hepatitis $\mathrm{B}$ surface antigen; AFP, alpha-fetoprotein; TNM, tumor-node-metastasis; MVI, microvascular invasion

Table 4 Univariate and multivariate analysis of overall survival (validation cohort, $n=247$ )

\begin{tabular}{|c|c|c|c|c|}
\hline \multirow[t]{2}{*}{ Clinical character } & \multicolumn{2}{|c|}{ Univariate analysis } & \multicolumn{2}{|c|}{ Multivariate analysis } \\
\hline & $\mathrm{HR}(95 \% \mathrm{Cl})$ & $p$ value & $\mathrm{HR}(95 \% \mathrm{Cl})$ & $p$ value \\
\hline AGLR level (>90 vs $\leq 90)$ & $2.47(1.59-3.64)$ & $<0.001$ & $1.82(1.35-2.57)$ & $<0.001$ \\
\hline Age, years (> 60 vs $\leq 60)$ & $1.16(0.77-1.76)$ & 0.460 & & \\
\hline Gender (male vs female) & $1.49(1.11-2.30)$ & 0.096 & & \\
\hline Drinking (yes vs no) & $1.23(0.88-1.71)$ & 0.207 & & \\
\hline HBsAg (positive vs negative) & $1.10(0.72-1.67)$ & 0.639 & & \\
\hline AFP, ng/ml (> 20 vs $\leq 20)$ & $1.65(1.13-2.65)$ & 0.011 & $1.07(0.81-1.44)$ & 0.366 \\
\hline Liver cirrhosis (yes vs no) & $1.03(0.59-1.79)$ & 0.801 & & \\
\hline Tumor size, cm (>5 vs $\leq 5)$ & $2.11(1.49-2.91)$ & $<0.001$ & $1.69(1.15-2.480)$ & 0.008 \\
\hline Tumor number (multiple vs single) & $1.63(1.22-2.54)$ & 0.013 & $1.20(0.81-1.79)$ & 0.307 \\
\hline TNM stage (III-IV vs I-II) & $2.33(1.83-3.50)$ & $<0.001$ & $1.39(1.07-2.10)$ & 0.016 \\
\hline MVI (yes vs no) & $2.81(1.99-3.73)$ & $<0.001$ & $2.30(1.57-2.93)$ & $<0.001$ \\
\hline Recurrence (yes vs no) & $2.51(1.64-3.51)$ & $<0.001$ & $2.05(1.46-2.79)$ & $<0.001$ \\
\hline
\end{tabular}

$\mathrm{Cl}$, confidence interval; $\mathrm{HR}$, hazard ratio; $\mathrm{AGLR}$, alkaline phosphatase plus gamma-glutamyl transpeptidase to lymphocyte ratio; $\mathrm{HBs} A g$, hepatitis $B$ surface antigen; AFP, alpha-fetoprotein; TNM, tumor-node-metastasis; MVI, microvascular invasion

cells by up-regulating the expression of metastasis related proteins [5], therefore, AFP was an unfavorable prognostic predictor. Tumor size is an important prognostic marker for $\mathrm{HCC}[3,19]$. Patients with a single tumor $>5 \mathrm{~cm}$ or multiple tumors would have a higher probability of bilobar involvement, invasion of microvascular and adjacent organs as well as the histologically positive margins [20]. MVI, which can lead to early postoperative recurrence and metastasis, is a significant risk factor of poor prognosis for $\mathrm{HCC}$ patients after radical resection as well as an independent predictor of long-term postoperative survival [21].
In recent years, many molecular biological models of liver cancer have been reported [22-24]. NLR and ALBI grades are simple and easy to determine preoperative predictors. In this study, we also analyzed the predictive effects of these two scoring models on the prognosis of liver cancer. The results are consistent with some previous studies [25-28], NLR and ALBI grade have predictive significance in $\mathrm{HCC}$ patients. Interestingly, we found that the predictive ability of AGLR was better than that of NLR and ALBI. Since the change of albumin level is related to the prognosis of HCC, Shen et al. proposed that albumin to gamma-glutamyltransferase ratio (AGR) 

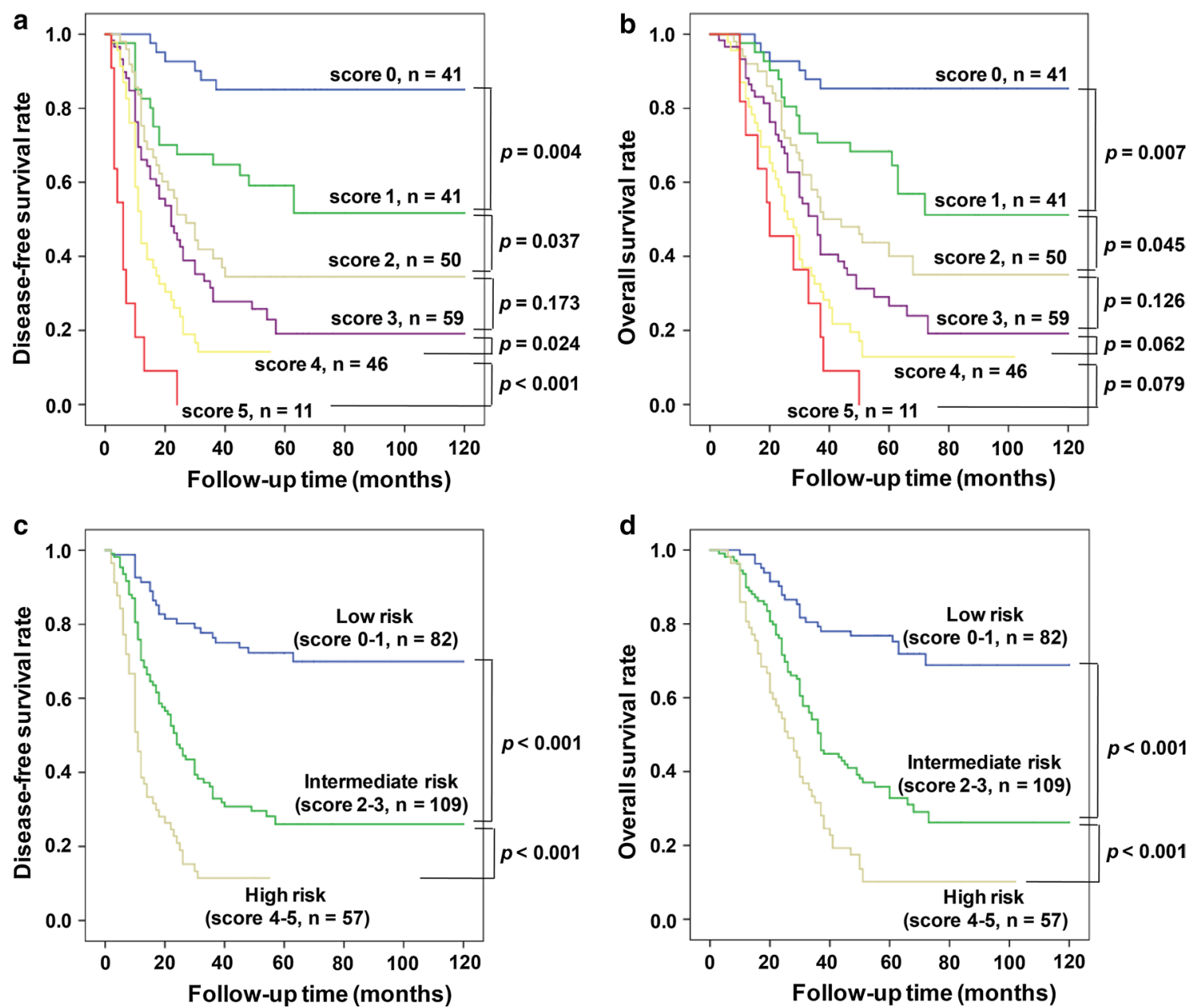

Fig. 5 In the training cohort, comparison of prognostic effects of different scoring groups, there was no statistical significance of survival between patients with a score of 2 vs. 3, for both DFS (a) $(p=0.173)$ and OS (b) $(p=0.126)$. Kaplan-Meier analysis of survival for different risks groups. There were statistical significance between the low-, medium- and high-risk groups (all $p<0.001$ ) for both DFS (c) and OS (d)

level might be related to the prognosis of HCC, and AGR classification was compared with platelet-to-lymphocyte ratio (PLR), it was found that the AGR-PLR score can further stratify HCC patients with different prognosis, and has a stronger predictive ability [29]. In this study, AGLR has the characteristics of simplicity and low cost, and it was confirmed that high levels of AGLR are associated with poor prognosis of HCC. In addition, we also found that AGLR level (>90), tumor size $>5 \mathrm{~cm}$, TNM stage III-IV, presence of MVI and recurrence were independent predictors of HCC survival by the multivariate analyses in both training and validation cohort. Considering the heterogeneity of prognosis, the predictive value of a single factor has certain of limitations, we established a simple prognostic scoring model based on the five independent predictors, which can be readily available in daily practice. All the $495 \mathrm{HCC}$ patients were randomly divided into the training cohort and validation cohort, and patients were further separated into the six different scoring groups. After that, we optimized this scoring model by changing the six scoring groups into the low-, medium- and high-risk groups, which could better predict different risks of survival. This new prognostic scoring model has potential application value in prognosis and can better predict the outcome of HCC patients. In the future, we will also consider comparing and merging AGLR with other prognostic scoring models to further improve the effect of predicting the prognosis of HCC patients and determine appropriate intervention.

There are some limitations in this study yet. Firstly, this is a retrospective study based on limited data of HCC patients from a single hospital, and only HCC patients accepted radical resection were enrolled in this study; thus, AGLR's prognostic prediction value for patients accepted liver transplant or TACE needs further study. Secondly, eastern and western countries' opinions on 

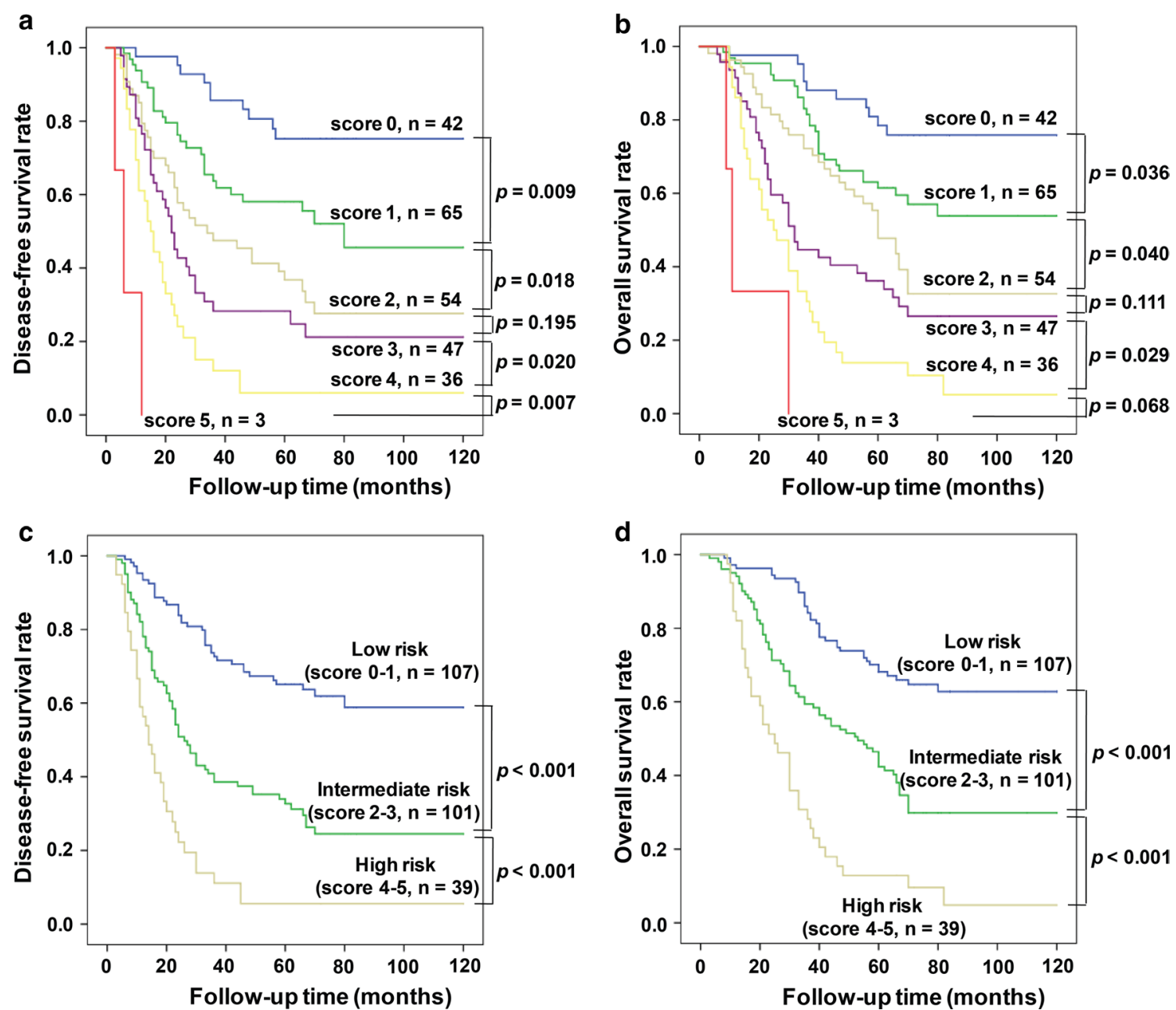

Fig. 6 In the validation cohort, DFS's patients with a score of 2 vs. $3(p=0.195)(\mathbf{a})$, OS's patients with a score of 2 vs. $3(p=0.111)$ and 4 vs. 5 $(p=0.068)(\mathbf{b})$ had no statistical significance. There were statistical significance between the low-, medium- and high-risk groups (all $p<0.001)$ for both DFS (c) and OS (d)

the surgical indications for $\mathrm{HCC}$ are still controversial [30], Third, the environmental background of HCC patients from different regions varies with each other. For instance, in China, the proportion of HBV-related HCC is nearly $90 \%$; whereas in western countries, most HCC are caused by alcoholic cirrhosis, non-alcoholic fatty liver disease and HCV infection [31]. Therefore, patients enrolled may suffer from obvious limitations of regional factors. For future researches, multicenter external validations and prospective studies are needed, so as to verify that this novel model may be widely available for HCC patients.

\section{Conclusions}

High preoperative AGLR level predicted poor prognosis for HCC patients; the simple and novel prognostic scoring model could effectively identify the higher risk of poor survival and early recurrence and may help select an appropriate treatment based on different risk stratification.

\section{Abbreviations}

HCC: Hepatocellular carcinoma; HBV: Hepatitis B virus; ALP: Alkaline phosphatase; GGT: Gamma-glutamyl transpeptidase; HBsAg: Hepatitis B surface antigen; AFP: Alpha-fetoprotein; NLR: Neutrophil-to-lymphocyte ratio; ALBI: Albumin bilirubin; AGR: Gamma-glutamyltransferase ratio; PLR: Platelet-to-lymphocyte ratio; TNM: Tumor node metastasis; MVI: Microvascular invasion; DFS: Disease-free survival; OS: Overall survival; Cl: Confidence interval; HR: Hazard ratio; ROC: Receiver operating characteristic;VS: Versus. 


\section{Acknowledgements \\ Not applicable.}

\section{Authors' contributions}

WL and RW designed the research; $J \mathrm{~L}$ and WL collected data; $Y L$ and RW performed the data analysis and model development; JY composed the first draft of the manuscript; RY commented on and critically revised the manuscript; $Y L, L Q$ and WL critically edited and reviewed the final draft of the manuscript. All the authors contributed to the conception of the study and approved the final manuscript.

\section{Funding}

This work was supported in part by the National Natural Science Foundation of China (No. 81372163), the National Key Sci-Tech Special Project of China (No. 2018ZX10302207), the Technology Planning Project of Guilin (No. 20190218-1) and Basic Ability Enhancement Program for Young and Middle-aged Teachers of Guilin Medical University (No. 2018glmcy073). The funding body had no role in the design of the study and collection, analysis, interpretation of data, or writing of the manuscript.

\section{Availability of data and materials}

The datasets used and/or analysed during the current study are available from the corresponding author on reasonable request.

\section{Ethics approval and consent to participate}

This study was approved by the research ethics committee of the Affiliated Hospital of Guilin Medical University and complied with the Declaration of Helsinki Principles. Written informed consent for treatment and use of their clinical data was obtained from all participants.

\section{Consent for publication}

Not applicable.

\section{Competing interests}

The authors declare that they have no competing interests.

\section{Author details}

${ }^{1}$ Laboratory of Hepatobiliary and Pancreatic Surgery, Affiliated Hospital of Guilin Medical University, Guilin 541001, Guangxi, People's Republic of China. ${ }^{2}$ Disease Prevention and Control Center of Guilin, Guilin, Guangxi, People's Republic of China. ${ }^{3}$ Department of Anesthesiology, The Second Affiliated Hospital of Guilin Medical University, Guilin 541001, Guangxi, People's Republic of China.

Received: 27 October 2020 Accepted: 27 December 2020

Published online: 03 February 2021

\section{References}

1. Siegel RL, Miller KD, Jemal A. Cancer statistics. CA Cancer J Clin. 2016;66:7-30

2. El-Serag HB. Hepatocellular carcinoma. N Engl J Med. 2011;365:1118-27.

3. Liu PH, Hsu CY, Hsia CY, Lee YH, Su CW, Huang YH, et al. Prognosis of hepatocellular carcinoma: Assessment of eleven staging systems. J Hepatol. 2016;64:601-8

4. Fu SJ, Zhao Q, Ji F, Chen MG, Wu LW, Ren QQ, et al. Elevated preoperative serum gamma-glutamyl transpeptidase predicts poor prognosis for hepatocellular carcinoma after liver transplantation. Sci Rep. 2016;6:28835.

5. Zhu Y, Xu D, Zhang Z, Dong J, Zhou Y, Zhang WW, et al. A new laboratorybased algorithm to predict microvascular invasion and survival in patients with hepatocellular carcinoma. Int J Surg. 2018;57:45-53.

6. Liao MJ, Qin WY, Liao Y, Yao RZ, Yu JX, Liao WJ. Prognostic value of gamma-glutamyl transpeptidase to lymphocyte count ratio in patients with single tumor size $<5 \mathrm{~cm}$ hepatocellular carcinoma after radical resection. Front Oncol. 2019;9:347.

7. Huang J, Liu FC, Li L, Yuan SX, Yang Y, Jiang BG, et al. Prognostic nomogram for hepatitis B Virus-related hepatocellular carcinoma with adjuvant transarterial chemoembolization after radical resection. Am J Clin Oncol. $2020 \cdot 43 \cdot 20-7$
8. Webber M, Krishnan A, Thomas NG, Cheung BM. Association between serum alkaline phosphatase and C-reactive protein in the United States National Health and Nutrition Examination Survey 2005-2006. Clin Chem Lab Med. 2010;48:167-73

9. Pike AF, Kramer NI, Blaauboer BJ, Seinen W, Brands R. A novel hypothesis for an alkaline phosphatase "rescue" mechanism in the hepatic acute phase immune response. Biochim Biophys Acta. 2013;1832:2044-56.

10. Kunutsor SK, Apekey TA, Seddoh D, Walley J. Liver enzymes and risk of all-cause mortality in general populations: a systematic review and metaanalysis. Int J Epidemiol. 2014;43:187-201.

11. Li G, Gao J, Tao YL, Xu BQ, Tu ZW, Liu ZG, et al. Increased pretreatment levels of serum LDH and ALP as poor prognostic factors for nasopharyngeal carcinoma. Chin J Cancer. 2012;31:197-206.

12. Clancy TE, Sengupta TP, Paulus J, Ahmed F, Duh MS, Kulke MH. Alkaline phosphatase predicts survival in patients with metastatic neuroendocrine tumors. Dig Dis Sci. 2006;51:877-84.

13. Ji F, Fu SJ, Guo ZY, Pang H, Ju WQ, Wang DP, et al. Prognostic value of combined preoperative lactate dehydrogenase and alkaline phosphatase levels in patients with resectable pancreatic ductal adenocarcinoma. Medicine (Baltimore). 2016:95:e4065.

14. Yamamoto K, Awogi T, Okuyama K, Takahashi N. Nuclear localization of alkaline phosphatase in cultured human cancer cells. Med Electron Microsc. 2003;36:47-51

15. Pratt DS, Kaplan MM. Evaluation of abnormal liver-enzyme results in asymptomatic patients. N Engl J Med. 2000;342:1266-71.

16. Jiao J, Zhao X, Hou R, Wang Y, Chang W, Liang N, et al. Comparison of two commonly used methods for stimulating T cells. Biotechnol Lett. 2019;41:1361-71.

17. Unitt E, Marshall A, Gelson W, Rushbrook SM, Davies S, Vowler SL, et al. Tumour lymphocytic infiltrate and recurrence of hepatocellular carcinoma following liver transplantation. J Hepatol. 2006:45:246-53.

18. Sideras K, Biermann K, Verheij J, Takkenberg BR, Mancham S, Hansen BE, et al. PD-L1, Galectin-9 and CD8 tumor-infiltrating lymphocytes are associated with survival in hepatocellular carcinoma. Oncoimmunology. 2017:6:e1273309

19. Xu XS, Wan Y, Song SD, Chen W, Miao RC, Zhou YY, et al. Model based on $Y$-glutamyl transferase and alkaline phosphatase for hepatocellular carcinoma prognosis. World J Gastroenterol. 2014;20:10944-52.

20. Lu Y, Zhu M, Li W, Lin B, Dong X, Chen Y, et al. Alpha fetoprotein plays a critical role in promoting metastasis of hepatocellular carcinoma cells. J Cell Mol Med. 2016;20:549-58.

21. Bruix J, Gores GJ, Mazzaferro V. Hepatocellular carcinoma: clinical frontiers and perspectives. Gut. 2014;63:844-55.

22. Mlecnik B, Tosolini M, Kirilovsky A, Berger A, Bindea G, Meatchi T, et al. Histopathologic-based prognostic factors of colorectal cancers are associated with the state of the local immune reaction. J Clin Oncol. 2011:29:610-8

23. Perry AM, Cardesa-Salzmann TM, Meyer PN, Colomo L, Smith LM, Fu K, et al. A new biologic prognostic model based on immunohistochemistry predicts survival in patients with diffuse large B-cell lymphoma. Blood. 2012;120:2290-6.

24. Lee JS, Heo J, Libbrecht L, Chu IS, Kaposi-Novak P, Calvisi DF, et al. A novel prognostic subtype of human hepatocellular carcinoma derived from hepatic progenitor cells. Nat Med. 2006;12:410-6.

25. Yang HJ, Guo Z, Yang YT, Jiang JH, Qi YP, Li JJ, et al. Blood neutrophillymphocyte ratio predicts survival after hepatectomy for hepatocellular carcinoma: A propensity score-based analysis. World J Gastroenterol. 2016:22:5088-95

26. Zheng J, Cai J, Li H, Zeng K, He L, Fu H, et al. Neutrophil to Lymphocyte Ratio and Platelet to Lymphocyte Ratio as Prognostic Predictors for Hepatocellular Carcinoma Patients with Various Treatments: a Meta-Analysis and Systematic Review. Cell Physiol Biochem. 2017:44:967-81.

27. Ho SY, Hsu CY, Liu PH, Hsia CY, Lei HJ, Huang YH, et al. Albumin-bilirubin grade-based nomogram of the BCLC system for personalized prognostic prediction in hepatocellular carcinoma. Liver Int. 2020;40:205-14.

28. Pan J, Chen S, Tian G, Jiang T. Preoperative Albumin-Bilirubin Grade With Prognostic Nutritional Index Predicts the Outcome of Patients With EarlyStage Hepatocellular Carcinoma After Percutaneous Radiofrequency Ablation. Front Med (Lausanne). 2020;7:584871. 
29. Shen J, Tang L, Zhang X, Peng W, Wen T, Li C, et al. A Novel Index in Hepatocellular Carcinoma Patients After Curative Hepatectomy: Albumin to Gamma-Glutamyltransferase Ratio (AGR). Front Oncol. 2019;9:817.

30. Berardi G, Morise Z, Sposito C, Igarashi K, Panetta V, Simonelli I, et al. Development of a nomogram to predict outcome after liver resection for hepatocellular carcinoma in Child-Pugh B cirrhosis. J Hepatol. 2020;72:75-84

31. Choo SP, Tan WL, Goh BKP, Tai WM, Zhu AX. Comparison of hepatocellular carcinoma in Eastern versus Western populations. Cancer. 2016;122:3430-46.

\section{Publisher's Note}

Springer Nature remains neutral with regard to jurisdictional claims in published maps and institutional affiliations.
Ready to submit your research? Choose BMC and benefit from:

- fast, convenient online submission

- thorough peer review by experienced researchers in your field

- rapid publication on acceptance

- support for research data, including large and complex data types

- gold Open Access which fosters wider collaboration and increased citations

- maximum visibility for your research: over 100M website views per year

At BMC, research is always in progress.

Learn more biomedcentral.com/submissions 